\title{
Informal psychiatric patients requesting discharge
}

\author{
Robin McGilp, Brian Kidd, Cameron Stark and Tom Henderson
}

\begin{abstract}
A retrospective investigation of case-notes compared 54 incidents of informal psychiatric in-patients being detained in hospital on an emergency basis with 66 incidents of discharge against medical advice (AMA). The characteristics of the two groups were compared. Detained patients were more likely to have been detained previousty, to be suffering from a psychotic illness, and to have threats of violence or self-harm mentioned in their case-notes. AMA patients were more likely to have a history of substance abuse but were no more likely than the detained group to have been discharged AMA in the past. The results suggest that psychiatrists in this hospltal are using current legistation on detention appropriately.
\end{abstract}

Psychiatrists are often asked to assess patients requesting yearly discharge from hospital. Those who are felt to require continuing in-patient treatment but who cannot be dissuaded from leaving may be either compulsorlly detained in hospital or discharged against medical advice (AMA). A number of previous studies have attempted to identify characteristics of either detained patients or those discharged AMA. Although both are possible outcomes when an informal patient requests discharge, there has been no work directly comparing the two groups.

Previous work on patients receiving irregular discharges is inconclusive. Rates vary widely between studies and definitions of what is classed as an irregular discharge are inconsistent. Hospital absconders are often included although they have been shown to have differing characteristics (Tomison, 1989). Clear criteria have therefore been suggested for defining the AMA discharge. (Dy, 1975).

In attempts to differentiate AMA patients from those receiving regular discharges, diagnostic and demographic variables have been examined (Philips \& Ali, 1983; Harper et al, 1982; Planasky \& Johnston, 1976; Miles et al. 1976). Although no clear pattern emerges, diagnoses of schizophrenia, personality disorder, to substance abuse, have been associated with AMA discharge. Younger age, male gender, and other variables such as time of discharge and grade of attending doctor have also been suggested as relevant.
Section 25 (1) of the Mental Health (Scotland) Act 1984, allows for the detention of informal patients already admitted to hospital if it is urgently necessary by reason of mental disorder for the health or safety of the patient or for the protection of other persons. Detention is for a maximum of 72 hours and requires a recommendation by one registered medical practitioner and if practicable, the consent of a relative or mental health officer. Uncomplicated alcohol or drug abuse are not considered adequate grounds for detention in themselves.

\section{The study}

The study was set in the acute psychiatric admission unit of an inner city general hospital serving a population of approximately 130000 . All patients discharging themselves AMA or detained under section 25 (1) between 1 January 1988 and 1 May 1991 were identified from hospital records. During the study period there were a total of 2010 discharges representing an AMA rate of $3 \%$.

AMA discharges were defined using Dy's guidelines, i.e. patients who requested to leave hospital, were seen by a doctor who did not regard them as detainable and when advised to remain, took their own discharge. Instances where patients had absconded, were persuaded to stay informally or were granted a regular but unplanned discharge were excluded.

Each request for discharge was treated as a discrete episode irrespective of outcome. It was therefore possible for patients to appear in the study more than once. Case-notes were examined retrospectively and information on three areas collected - previous history, current admission and the request for discharge (see Table 1).

ICD-9 diagnoses were take from SMR4 recording forms and were then sub-classified as 'psychotic' and 'non-psychotic' as in previous studies. Psychotic illness included manic depressive psychosis, schizophrenia, paranoid psychosis, schizoaffective disorder, delirium tremens, and drug-induced psychosis. Substance abuse included alcohol or drugs. 
Table 1. Information collected from case-notes

\begin{tabular}{lll}
\hline Previous history & Current admission & Request \\
\hline Age & Length of stay & Time of request \\
Sex & Ward & Grade of doctor \\
Previous diagnosis & Responsible medical officer & Threats of violence \\
Number of admissions & DSH during this admission? & Threats of DSH \\
History of substance abuse? & Violence during this admission? & \\
History of DSH?* & ICD-9 diagnosis & \\
History of violence? & & \\
Previously detained? & & \\
Previously discharged AMA? & & \\
\hline
\end{tabular}

"Deliberate self harm (DSH) included self poisoning and self injury.

Table 2. Characteristics of groups detained or discharged AMA

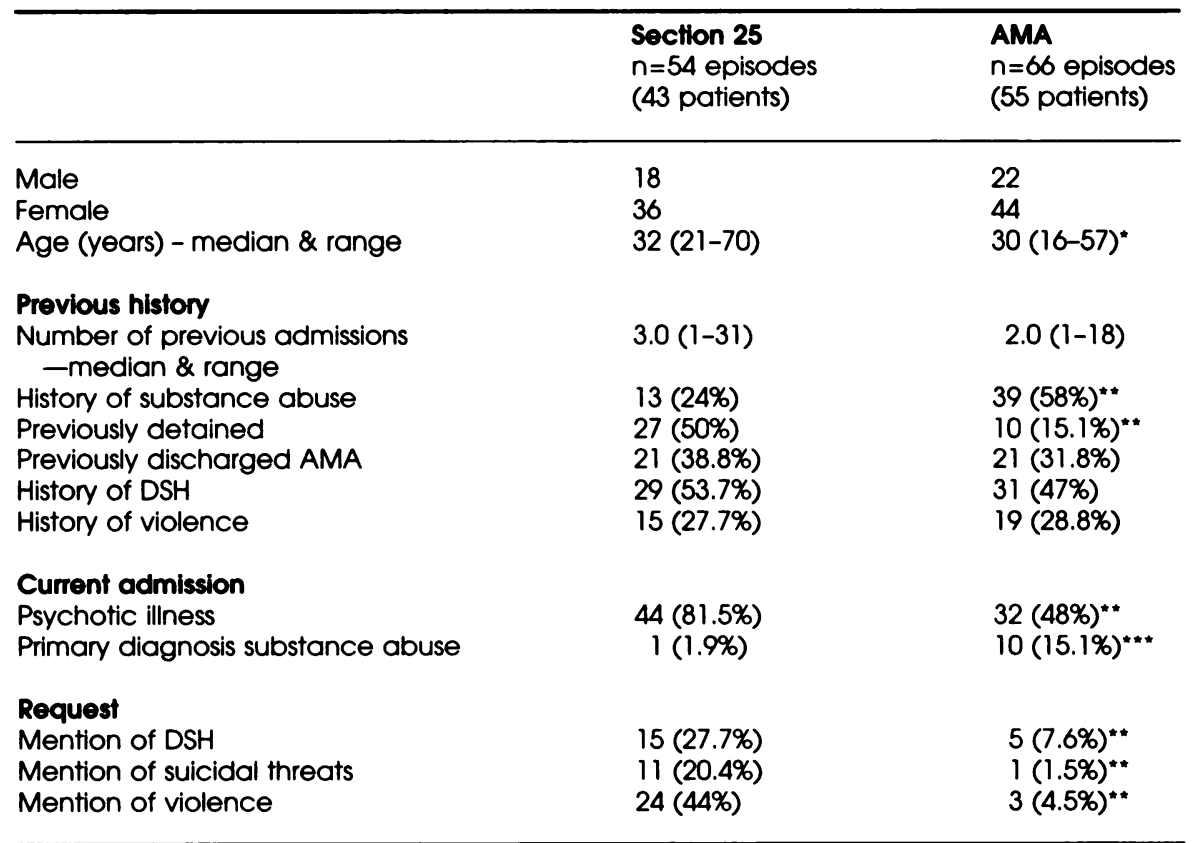

"= not significant by Mann-Whitney $U$ test.

${ }^{* *}=P<0.01$ by $\chi^{2}$ test with Yates' correction.

$* * * P<0.05$ by $\chi^{2}$ test with Yates' correction.

\section{Findings}

Table 2 shows the results - only significant differences between the two groups are presented. The two groups were similar in age and sex distribution. In their previous histories there were no significant differences in number of admissions, previous diagnosis of personality disorder, history of AMA discharge, deliberate self-harm (DSH) or violence. Differences in time of day, seniority of doctor involved, ward or clinical team did not achieve significance.

A number of factors in the previous history or current admission distinguished the two groups. Detained patients were significantly more likely to have been detained during a previous admission, to have a current diagnosis of psychotic illness, and to have DSH, suicidal threats, or violence mentioned in the relevant entry in their case-notes. AMA patients were significantly more likely to have a previous 
history or current diagnosis of substance abuse.

\section{Comments}

The low AMA rate in comparison with other studies is probably due to our use of a much tighter definition and reflects a 'true' rate of self discharge against medical advice. It is surprising that there is a clear excess of females in each group as other work suggests that both AMA and detained patients tend to be men. In this study, patients demanding an unplanned discharge were twice as likely to be women. The reasons for this are not apparent from the results. Between the two groups there are no significant difference in median age and in spite of a wide range of ages, most requests tended to come from younger patients. Having a current diagnosis of drug and alcohol abuse or a previous history of the same emerge as the two distinguishing features of those discharged AMA. Although 58\% of the AMA group had a history of substance abuse (twice as common as in the detained group), it was the current primary diagnosis in only $15 \%$. Those in other diagnostic groups were more likely to be discharged AMA if they had a history of previous substance abuse. It seems that a history of substance abuse influences the decision to discharge AMA independent of the current diagnosis. This confirms the findings of other studies and may reflect the impulsive nature of this group of patients and the difficulties of caring for them in conventional psychiatric wards.

Of section 25 patients, $50 \%$ had previously been detained compared with only $15 \%$ of AMAs. This is partly due to the recurrent nature of chronic psychotic illness. It could also be argued that a previous history of detention influences the doctor and increases the probability of subsequent detention, i.e. that decisions are made on the basis of what was done the last time a similar situation arose. The finding that the section 25 patients were as likely as the AMA patients to have been discharged AMA in the past suggests that this is not the case. It is also noteworthy that a previous history of violence or DSH did not appear to influence the decision.

Regarding the current admission, a current diagnosis of psychotic illness was significantly more frequent in the detained group. This rather crude method of dividing diagnoses into 'psychotic' and 'non-psychotic' was taken from other studies and does not allow more detailed examination of patterns.

Detained patients were also more likely to have threats of violence or deliberate self-harm recorded in the relevant entry in the case-notes.
This may suggest that current behaviour at the time of the request has a greater influence than features in the previous history. It is of course also possible that doctors may be less likely to record such details in the case-notes of those patients they have decided to allow to go AMA. The finding that the seniority of the doctor concerned, ward, responsible medical officer, and time of day were not significantly different in either group suggests that the patient's illness and current clinical state were more important than the setting or circumstances of the request.

There are significant differences in the characteristics of the two groups studied. They were distinguished by those factors related to the current episode, i.e. psychotic illness and behaviours dangerous to the patient or other people. It is also of note that detained patients had frequently been detained previously. In making the decision to detain or to discharge AMA doctors in the hospital studied appear to be acting within the terms of the current legislation and are not unduly influenced by previous decisions made under similar circumstances.

\section{Acknowledgement}

Our thanks go to Jennifer Ranger and Sheila McTaggart for their help in this study.

\section{References}

DY, A.J. (1975) Defining the AMA discharge. (letter) American Journal of Psychiatry. 132, 297.

HARPER, D.W., ElLIOT-HARPER, C., WEINERMAN, R., ANDERSON. N.J. \& NELSON. T.A. (1982) Comparison of AMA and Non-AMA Patients on a Short-Term Crisis Unit. Hospital and Community Psychiatry. 33, 46-48.

Miles, J.E., Adelsberg, M.. Rerth. G. \& Cumming. J. (1976) Discharges against medical advice from voluntary psychiatric units. Hospital and Community Psychiatry. 27. 859-865.

PHILliPS, M.S. \& ALl. H. (1983) Psychiatric patients who discharge themselves against medical advice. Canadian Journal of Psychiatry. 28, 202-205.

Planasky, K. \& Johnston. R. (1976) A survey of patients leaving hospital against medical advice. Hospital and Community Psychiatry, 27, 865-868.

Tomison. A. (1989) Characteristics of psychtatric hospital offenders. British Journal of Psychiatry. 164, 368-371.

* Robin McGilp, Senior Registrar in Psychiatry, Royal Dundee Liff Hospital, Dundee DD2 5NF; Brian Kidd, Registrar in Psychiatry, Southern General Hospital, Glasgow G51 4TF; Cameron Stark, Registrar in Public Health, Argyll and Clyde Health Board; and Tom Henderson, Registrar in Psychiatry, Bellsdkye Hospital, Falkirk FK5 4SF

*Correspondence 